\title{
13 STEP SUPPORT FOR VIRTUAL ENTERPRISES
}

\author{
Andreas Schreiber ${ }^{\star}$ \\ ProSTEP GmbH, Germany
}

\begin{abstract}
Product information is an essential production factor for virtual enterprises especially in all design and manufacturing processes. The efficient use of this production factor can be measured by its availability. The technology that is used in order to make information available is the information technology. With respect to the technical processes used in design and production the information technology is commonly referred to as product data technology (PDT). This chapter describes how a STEP based PDT concept can be used to increase the enterprises' efficiency in the design phase within a Virtual Enterprise.
\end{abstract}

\section{INTRODUCTION}

\section{The need of PDT}

The major goal of PDT (Product Data Technology) is to assure the overall availability, reusability and processing capability of product information described by means of information processing systems, which, in particular, has a decisive effect on both the economy and company flexibility, and therefore ultimately the competitiveness of a company. In order to support close partnerships in virtual enterprise environments, the ability of CAx, PDM (Product Data Management) and PPC systems to communicate must be forcefully promoted as the basis of measures that increase efficiency, such as simultaneous engineering or the support of closer partnerships between manufacturers and suppliers. Communication in this context refers not only to the exchange of information between the different systems, but rather to the ability to access, interpret and subsequently process the information across the boundaries of systems and companies throughout the entire computeraided process in the development, design, fabrication, maintenance, and disposal of products and prodution facilities.

\footnotetext{
- ProSTEP GmbH und Verein, Julius-Reiber-Straße 15, 64293 Darmstadt, Germany, Tel.: 06151-9287-0, Fax: 06151-9287-26, e-mail: schreiber@prostep.de
}

The original version of this chapter was revised: The copyright line was incorrect. This has been corrected. The Erratum to this chapter is available at DOI: 10.1007/978-0-387-35577-1_37 


\section{DISTRIBUTED PRODUCT DEVELOPMENT}

\section{Needed information in Distributed Product Development Processes}

The development of a PDT concept requires an analysis in order to identify the systems and types of information that will be involved in development and production processes. To define the product data which have to be managed by the PRODNET architecture an analysis was undertaken, which

- identified product data needed in virtual enterprise environments, and

- connected these data with the responsible type of system.

In development and production processes different types of information are needed:

- part master data (identification, description, name, ...)

- product configuration

- product structure (assembly, component relationships, ...)

- document (all kinds of documentation)

- document structure (relationships between different documents)

- properties (material, size, quantity, mass, ...)

- classification

- work management (approval, change management, ...)

- geometry, topology

- surface conditions, form features

- financial and logistic data (order data, sales information, ...)

In order to manage the above mentioned information in development and production processes different types of systems are used (ECAD, MCAD, CAE, CAP, CAQ, PDM, BoM-Systems, TDM, PPC, ...). In order to focus the paper to requirements of small and medium sized companies, CAD, PDM and PPC systems are mainly discussed in the following chapters.

Some types of information are managed by more than one system (for example the product structure is managed by the PDM system as well as by the PPC system). For that reason a smooth communication is required between systems of different types, too. Besides this circumstance some additional problems arise in the communication of product data between companies in a virtual enterprise environment.

\section{Need of data exchange}

For the generation, processing and management of data describing complex products and systems like a car or fitness equipment many different computer-aided systems are currently in use. In the context of computer-integrated production, the exchange of these product defining data plays an increasingly important role. Figure 1 shows the necessity of close communication and coordination between different development departments as well as between cooperation partners in the field of automotive industry. In the production of fitness equipment, which is the subject of 
PRODNET II, the same requirements for communication between different departments as well as between the manufacturer (Orbita) and the supplier (Miralago), can be found.

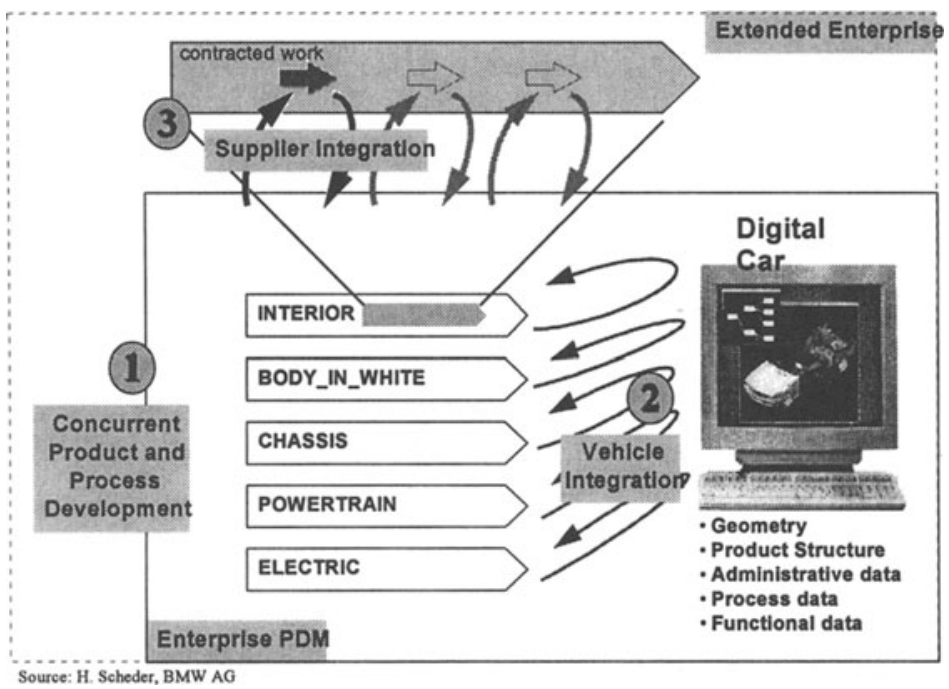

Figure 1 - Distributed product development processes in the automotive industry (source: BMW)

Today the mentioned data are generated and processed by CAD/CAM systems in predominantly incompatible formats and structures. The integration of data is a prerequisite for concurrent engineering. For instance, design data can only be reused efficiently in downstream departments (e.g. FE analysis or NC manufacturing) if the data is already available as CA models and if it is not necessary to regenerate these data in CA systems by using drawing information and manual input. Reusing the data decreases the risk of errors on input and reduces the overall effort. The exchange of product model data is significant to companies for their internal and external data exchange between information processing systems. The aim of internal product data exchange is to provide information technology links between the various departments of a company such as design, project planning, manufacturing, assembly and quality assurance. The external product data exchange is used for transmitting product information between manufacturers and suppliers.

The product structure, for example, is managed in three different systems: the CAD system, the PDM system in which the CAD models and drawings are managed and, of course, in the bill of materials (BoM) and the PPC system. Each user has his particular view of the product structure. The designer, for example, defines it from a functional point of view, in the PDM system it is administered from the viewpoint of product documentation, while the PPC system handles it from the manufacturing point of view, enriched with a lot of additional information. At present, the design engineer cannot access the data in the BoM system directly from his CAD 
workstation. He has to change the application system and spend valuable time entering the data again or duplicating it. Safeguarding the consistency of data today is only possible at a great expense and is often only possible by manual means (Figure 2).

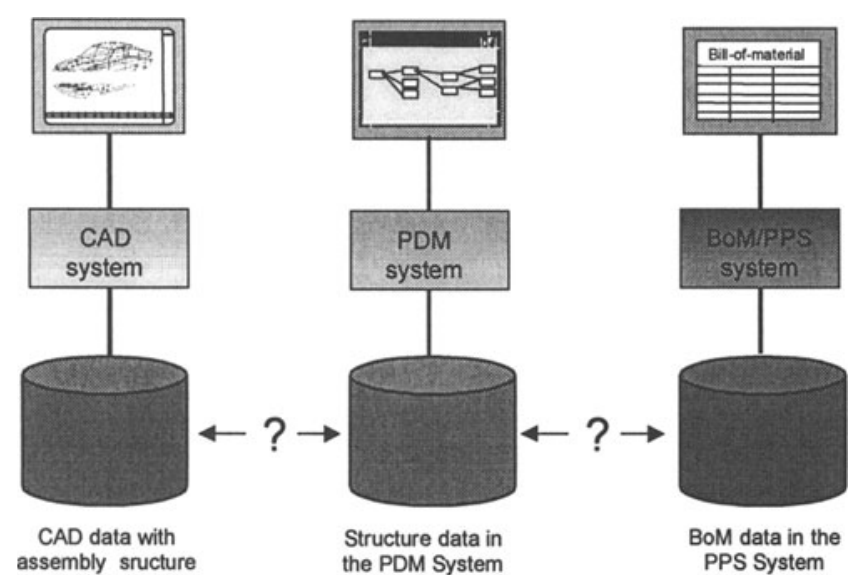

Figure 2 - Current situation: Isolated systems with incompatible and redundant data

An extremely important aspect is the cooperation with suppliers in the computeraided product development. Today it is no longer sufficient to exchange geometry of components. The development partners must work in a common context and to this end be able to access consistent data on the structure, release and project. They generally work as a team that crosses company boundaries and each member requires direct access to the latest status of the development data.

A prerequisite for an integrated environment is the availability of neutral interfaces for the exchange of product data. Such an interface is defined by the product data standard STEP.

\section{ISO 10303 (STEP)}

The Standard for the Exchange of Product Model Data (STEP - ISO 10303) aims to manage all product-related data during the whole life cycle of the product. The benefits that can be obtained from almost all STEP applications, include:

- system independence,

- data consistency across the entire product life cycle,

- redundancy-free product data description,

- higher quality of the product data due to unambiguous definitions and therefore higher quality of product data exchange, and

- possibilities of long-term archiving. 


\section{STEP APPLICATION PROTOCOLS}

Different processes cause deviations in the product model of different industrial branches. One example is the deviations in the product documentation data of production processes of single units and continuous production processes. In the context of mechanical products other objects for product description are important than for electronic products (e.g. printed circuits). Therefore application protocols (ISO 10303-201) have been standardized in STEP to define such application-specific product data models.

As a result of collaboration among the German Automotive Association (VDA), ProSTEP and other international partners, the application protocol Core Data for Automotive Mechanical Design Processes is currently being developed as ISO standard 10303-214. The Application Protocol ISO 10303-214 describes the product and production facilities data of development and supply process chains in several industries.

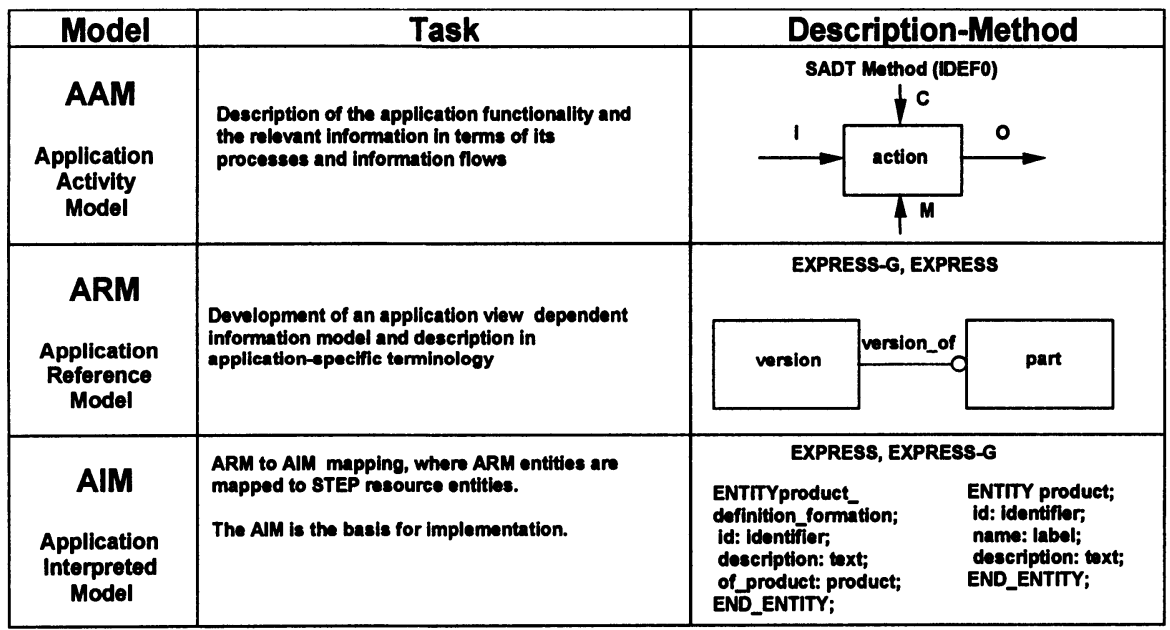

Figure 3 - Components of an application protocol

The application protocol 212 Electrotechnical Design and Installation is an initiative of the electrotechnical and automotive industry. It is being developed by ProSTEP and Siemens in collaboration with DKE and other international partners and will be standardized as the ISO/IEC standard 10303-212 within the framework of the joint activities of ISO TC184/SC4 and IEC TC3. The AP212 is a solution for the representation of electrotechnical product characteristics. It describes the product data of development process chains in the electrotechnical industry.

An application protocol consists of the following parts (Figure 3):

1. Functional model of the applications (Application Activity Model, AAM) that shall be supported by the application protocol. The AAM specifies the scope of the standard and is defined using the SADT method (IDEF0). The AAM 
identifies those data classes that are input, output, control and method elements of the considered functions / activities.

2. The product data model from an application perspective (Application Reference Model, ARM) specifies the data classes identified in the AAM using a formal description method. EXPRESS-G is normally used for this task.

3. The third main part (Application Interpreted Model, AIM) contains the description of the application-specific ARM using the predefined building blocks of the integrated resources (generic data model that can be used by all APs). For this purpose a mapping between the objects in ARM and AIM is defined.

\section{USING THE PDM SCHEMA IN THE PRODNET PROJECT}

With the development of the STEP application protocol AP214, the international industry partners are aiming to use a standardized description of data to improve the supply of information in the development process chains, despite heterogeneous system environments.

But besides the AP214 for mechanical information other sectors have defined different application protocols, such as the AP203 that has been defined on the basis of the requirements of the American aerospace industry and of the MIL standards of the US Department of Defense. A further example is the AP212 initiated by the electrical industry.

As the requirements are specific to individual industries, different data models arise at ARM-level even within the same subject areas. Although a mandatory and generic set of basic constructs is available for the definition of the AIM, the interpretation is AP-specific.

Users and system vendors, however, expect coordinated specifications [MACH, 98]:

- The user, in order to be able to use data models in cooperation.

- The system vendor, in order to be able to offer standard software to the entire market.

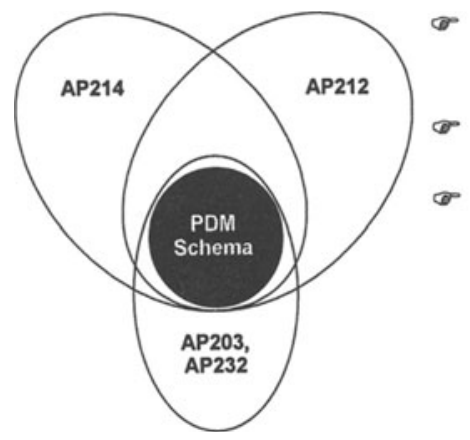

CommonPDM data schema generated and maintained by PDES, Inc., ProSTEP andJSTEP

Real Subset ofPDM relevant STEP APs (AP203, 212, 214, 232)

Fulfills nearlyall requirements for PDM data exchange Main functionality for parts and documents:

- identification

- versioning

- structures incl . transformations

- approvals and authorization

- project, work order, work request

- effectivities

- classification and properties

Figure 4 - PDM Schema as intersection between different application protocols 
For this reason, ProSTEP and PDES, Inc. have been devoted to the subject of AP interoperability ([STAUB, 97]). The most important result of this work is the PDM schema. This arose as a result of the coordination of the application protocols AP203, AP212, AP214 and AP232 relevant to the manufacturing industry in the field of organizational product data. The first version of the schema was published jointly by ProSTEP, PDES, Inc. and the Japanese STEP organization JSTEP in 1997 ([ProSTEP, 97]).

The PDM schema is a data model at AIM level. It is the universal coordinated specification for the exchange of organizational product data by means of physical file data exchange.

The PDM schema supports data for the identification and structuring of products and documents, for the description of properties, of attributes for the authorization and management of modification, and development jobs and requests. Figure 5 shows the current scope of the PDM schema.

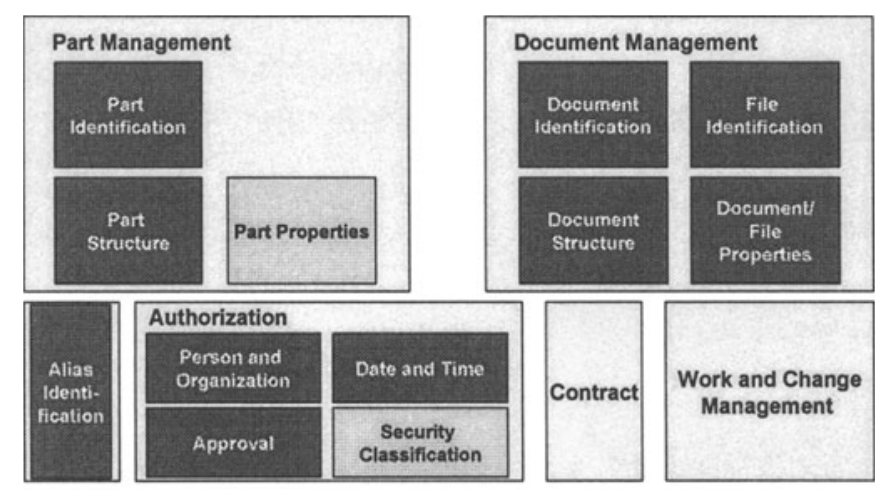

Figure 5 - PDM data classes

\section{PROSTEP PRODUCTS USED IN PRODNET}

In order to make STEP technology available to SME's participating in PRODNET II two product data processing tools were integrated. The STEP module acts as a server for functionality's provided by these tools.

The PDM Editor is a tool for viewing and editing of organizational product information (part master data, product structure, document structure, approval ...). The PDM Editor is able to access STEP information based on the PDM Schema specification.

IntraVISION is a graphical viewer for visualizing geometric product information created by CAD systems. The product enables a wide variety of neutral formats to be displayed. The formats supported include 2D/3D geometry formats such as STEP, IGES and VDAFS, plotter formats such as HPGL and CGM as well as bitmap and documentation formats such as TIFF, CALS Raster and PDF. 


\section{THE PRODNET II CASE}

In order to prove the usability of the PDT approach an application case is needed. For SME's the digital exchange of product data between manufacturer and supplier gains importance. The following scenario outlines such a communication in the field of part master data.

A new version of a part has to be developed by a supplier. The manufacturer therefore sends the data for the original version, the organizational data for the new version, and the documents describing the original version to the supplier. The manufacturer expects to receive the documentation for the new version of the part from the supplier.

In the manufacturer's PDM system, the documents for the existing version, the organizational data for the old and new versions, and the data for the documents are selected. The organizational data is written to a STEP physical file by the PDM STEP processor.

The EDI module inside the PRODNET architecture at the manufacturer's site packs the PDM physical file and the files of the documents (e.g. DXF file containing 3D geometry in an EDIFACT/CONDRA envelope) and sends it to the supplier.

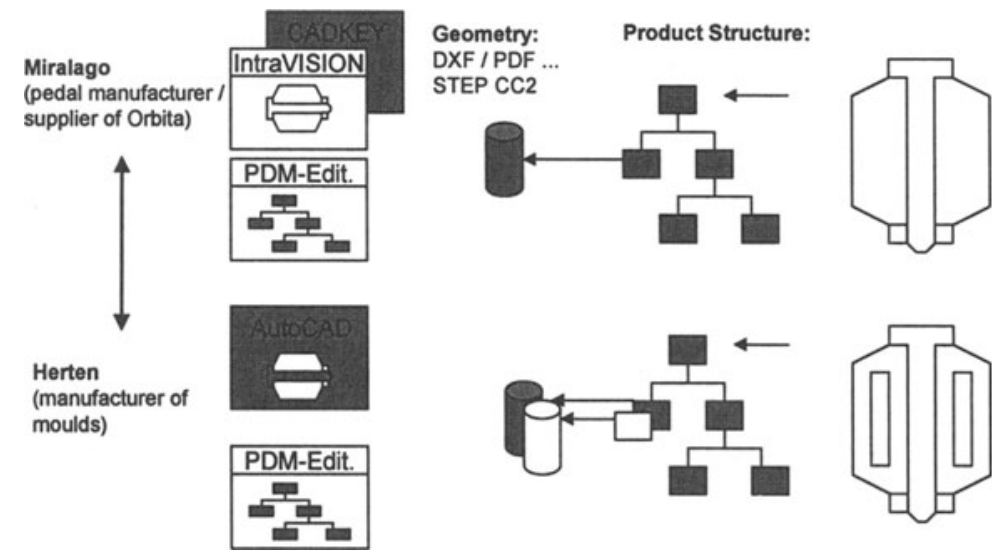

Figure 6 - Product data exchange between Miralago and Herten

The EDI module at the supplier's site unpacks the PDM file and the files containing the documents. The PDM Editor for the organizational data permits input control for the organizational data of the parts and documents. IntraVISION permits inspection of 3D geometry and drawings.

In the communication between Miralogo and Herten we can find both, the exchange of geometric information as well as the exchange of organizational information ( Figure 6). Miralago acts as subcontractor of Orbita who provides parts of fitness equipment like bike pedals. Herten acts as toolmaker that supplies injection moulds for the production of the pedal body.

In the scenario described above not only product data are needed. The 
PRODNET architecture aims a consistent exchange of commercial information (e.g. offer, order, and invoice) and product data (product structure, geometry) to guarantee close development relationships in virtual enterprise environments. To achieve this goal the STEP module has to provide product data related services, which are accessible by all components integrated in PRODNET II.

\section{STEP MODULE ARCHITECTURE}

The STEP module provides services for accessing STEP files and acts as an interface to the mentioned ProSTEP products PDM Editor and IntraVISION. This module enables PRODNET II to generate and process product data based on the neutral standard STEP.

\section{Export STEP information}

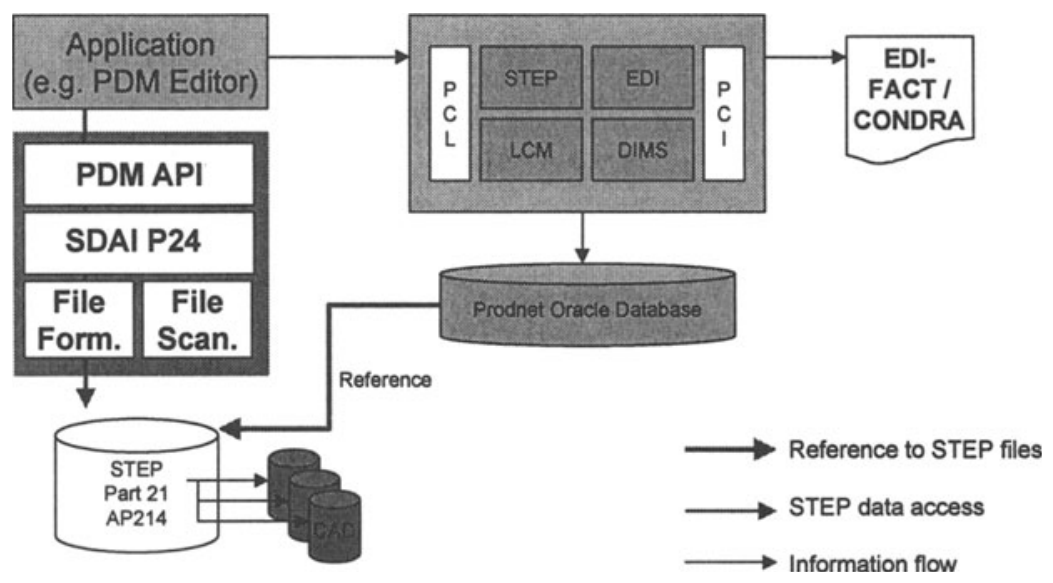

Figure 7 - PRODNET Architecture for STEP export

The design engineer uses the PDM Editor for creating part master data related to an assembly. He is able to define the product structure, and create parts and versions. After that he creates the appropriate document structure and connects already produced CAD models to this structure. By pushing a send button, the created PDM STEP information as well as the CAD model information is transferred to the PRODNET STEP module (Figure 7). This module stores all needed information in the DIMS database and calls the service for sending all files with an EDIFACT/CONDRA message.

\section{Import STEP Information}

Figure 8 shows how to process product data management information organized in a 
STEP physical file. After receiving an EDIFACT/CONDRA message with attached CAD and PDM files the STEP module is activated. The service for importing product data access organizational data in the DIMS database. After that the PDM Editor is called with the specified PDM file name. The PDM Editor signals the design engineer import activities that opens the PDM Editor in order to validate the received information.

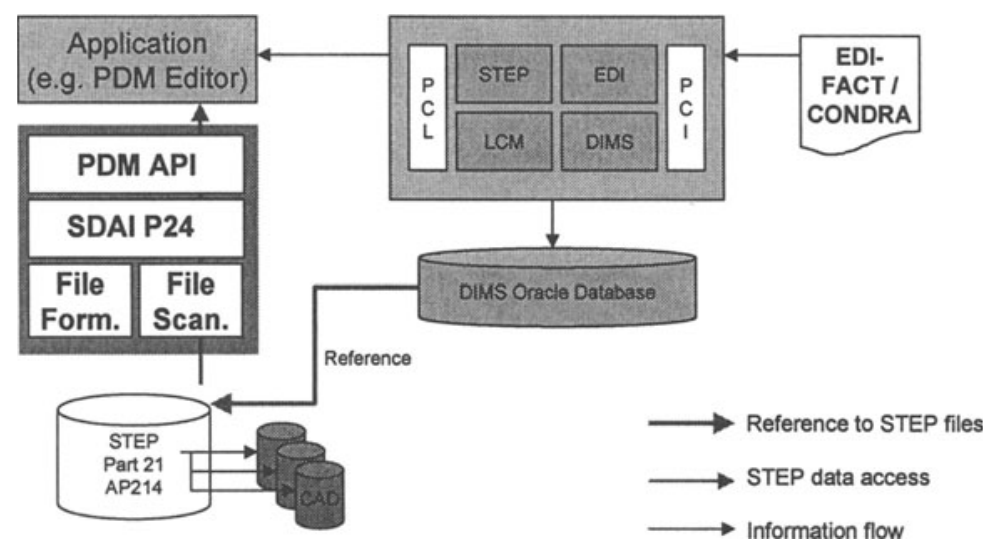

Figure 8 - PRODNET Architecture for STEP import

\section{SUMMARY AND PROSPECTS}

The PRODNET product data concept aims to improve the capabilities of SME's in the field of digital product data exchange. Strong customers increasingly force these companies to process and to supply product data like part master data or geometry. The discussed concept makes available product data technology on the base of neutral standards using low price products (PDM Tools and Geometry Viewer instead PDM and CAD systems). Therefore the companies can use the same approach in the communication with different partners.

In PRODNET the PDT concept was proved as usable for SME's in the discussed scenario. Nevertheless there are still some open tasks. Especially a closer integration of product data beyond the scope of single systems (like PDM or PPC) is needed. Additionally the electronic data exchange on the basis of EDIFACT has to be discussed regarding competing standards like Odette, VDA or ANSI X.12.

\section{REFERENCES}

1. Machner, B.; Ungerer, M.: Mapping the User Requirements from the VDAORG with the PDM Schema. ProductDataJournal 5 (1998) 1

2. ProSTEP, PDES, Inc., and JSTEP Announce PDM Schema. ProductDataJournal 4 (1997) 2

3. Staub, G.: Harmonization of the application protocols AP212 and AP214. ProductDataJournal 4 (1997) 2 